\title{
Photohadronic Processes in Astrophysical Environments
}

\author{
A. Mücke ${ }^{1}$, J. P. Rachen ${ }^{2}$, Ralph Engel $^{3}$, \\ R. J. Protheroe ${ }^{1}$ and Todor Stanev ${ }^{3}$ \\ ${ }^{1}$ Department of Physics and Mathematical Physics, University of Adelaide \\ Adelaide, SA 5005, Australia. \\ amuecke@physics.adelaide.edu.au, rprother@physics.adelaide.edu.au \\ ${ }^{2}$ Department of Astronomy, Pennsylvania State University, \\ University Park, PA 16802, USA \\ jorg@astro.psu.edu \\ ${ }^{3}$ Bartol Research Institute, University of Delaware, \\ Newark, DE 19716, USA \\ eng@lepton.bartol.udel.edu, stanev@bartol.udel.edu \\ Received 1998 September 3, accepted 1999 April 20
}

\begin{abstract}
We discuss the first applications of our newly developed Monte Carlo event generator SOPHIA to multiparticle photoproduction of relativistic protons with thermal and power-law radiation fields. The measured total cross section is reproduced in terms of excitation and decay of baryon resonances, direct pion production, diffractive scattering, and non-diffractive multiparticle production. Non-diffractive multiparticle production is described using a string fragmentation model. We demonstrate that the widely used ' $\Delta$-approximation' for the photoproduction cross section is reasonable only for a restricted set of astrophysical applications. The relevance of this result for cosmic ray propagation through the microwave background and hadronic models of active galactic nuclei and gamma-ray bursts is briefly discussed.
\end{abstract}

Keywords: nuclear reactions_elementary particles_radiation mechanisms: non-thermal

\section{Introduction}

The energy spectrum of cosmic rays extends to energies above $10^{20} \mathrm{eV}$. Such particles are most likely extragalactic and propagate through many tens of Mpc before reaching the Earth. The main energy loss mechanism for nucleons in this energy range is photomeson production on the cosmic microwave background radiation (CMBR). This process has an energy threshold of 1.08 $\mathrm{GeV}$ in the centre of momentum frame (CMF) of the interacting particles. It causes the distortion of the proton spectrum above $3 \times 10^{19} \mathrm{eV}$ during propagation, known as the Greisen-Zatsepin-Kuzmin cutoff (Greisen 1966; Zatsepin \& Kuzmin 1966; see Protheroe \& Johnson 1996 for additional references). In environments harbouring dense radiation fields with higher photon energies, the particle production threshold is reached at lower proton energies, e.g. $\sim 10^{16} \mathrm{eV}$ for ambient photon energies of $10 \mathrm{eV}$. If protons are accelerated in such energetic astrophysical objects, photopion production and subsequent pion decay would lead to the emission of energetic $\gamma$-rays and neutrinos (see Berezinsky \& Gazizov 1993a,b for neutrino production in $N \gamma$ scattering). This may be observable from jets in active galactic nuclei (AGN) (Mannheim 1993; Protheroe 1997) and gamma-ray bursts (GRB) (Waxmann \& Bahcall 1997; Vietri 1998a,b; Böttcher \& Dermer 1998; Rachen \& Meszaros 1998).

The prominent $\Delta(1232)$-resonance near the threshold has often been used to construct approximate pion production cross sections (referred to as the ' $\Delta$-approximation' hereafter) and to determine gross features like $\gamma$-ray-to-neutrino energy yields, proton inelasticities, etc. that enter the relevant astrophysical calculations. In this paper we discuss the first results obtained using a new Monte Carlo event generator for photohadronic interactions of relativistic protons in radiation fields of astrophysical origin, which includes all relevant interaction processes and is based on models and data available from particle physics.


$\underline{P h o t o-} \underline{H}$ adronic $\underline{I}$ nteractions in $\underline{A}$ strophysics), has been extensively tested on all fixed target and collider experiment data available to us (Mücke et al. 1999a). A comparison of the results from sopHIA with the $\Delta$-approximation emphasises the importance of including all interaction processes in calculations of expected astrophysical signals. Detailed estimates of such signals will be the subject of future work. This paper concentrates on photopion production 


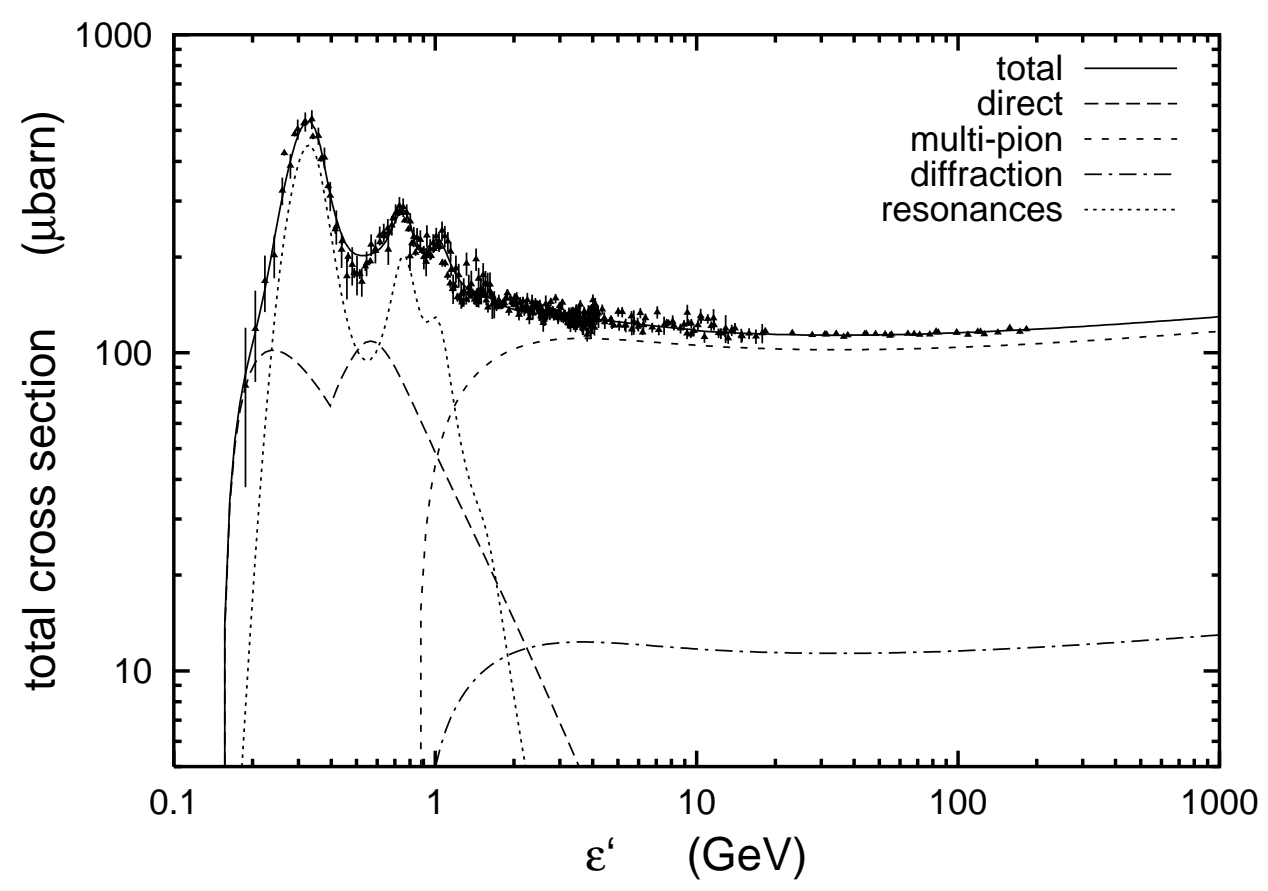

Figure 1-The total $p \gamma$ cross section, with the contributions of the baryon resonances considered in this work, the direct single-pion production, diffractive scattering, and the multipion production as a function of the photon's NRF energy $\left(1 \mu\right.$ barn $\left.=10^{-34} \mathrm{~m}^{2}\right)$. Data are from Baldini et al. (1988).

features that may be important for astrophysical processes which involve hadronic reactions. In Section 2 we summarise the physics implemented in sophis and contrast it with the $\Delta$-resonance approximation. Various astrophysical applications of SOPHIA are discussed in Section 3 by considering power-law and thermal photon seed spectra. A summary is given in Section 4.

\section{Cross Section and Kinematics}

Consider a relativistic proton with energy $E_{p}=\gamma m_{p}$ and rest mass $m_{p}$, which interacts with a photon of energy $\epsilon$ at an angle $\theta$. The square of the total $\mathrm{CMF}$ energy of the interaction is given by

$$
s=m_{p}^{2}+2 E_{p} \epsilon(1-\beta \cos \theta)=m_{p}^{2}+2 m_{p} \epsilon^{\prime},
$$

where $\beta=\sqrt{1-\gamma^{-2}}$ is the velocity of the proton in terms of the velocity of light, and $\epsilon^{\prime}$ is the photon energy in the nucleon rest frame (NRF). In SOPHIA, proton- and neutron-induced interactions are distinguished; for simplicity we quote only proton interactions in the following.

The partial cross sections for resonance excitation, direct (non-resonant) single-pion production, and diffractive scattering are determined by fits to exclusive data (Figure 1). We consider the nine most important resonances $\left[\Delta^{+}(1232), N^{+}(1440)\right.$,
$N^{+}(1520), \quad N^{+}(1535), \quad N^{+}(1650), \quad N^{+}(1680)$, $\Delta^{+}(1700), \Delta^{+}(1905)$ and $\left.\Delta^{+}(1950)\right]^{1}$, and use the Breit-Wigner formula together with their known properties of mass, width and decay branching ratios to determine their contributions to the individual interaction channels. The direct channel is defined as the residual, non-resonant contribution to the channels $p \gamma \rightarrow n \pi^{+}, p \gamma \rightarrow \Delta^{++} \pi^{-}$, and $p \gamma \rightarrow \Delta^{0} \pi^{+}$. The method is described in detail in Rachen (1996). Note that although the $\Delta(1232)$-resonance has the highest cross section at low interaction energies, the direct channel dominates near threshold. The direct channel can be important for proton interactions in soft photon spectra since it produces exclusively charged pions. Diffractive scattering is due to the coupling of the photon to the vector mesons $\rho^{0}$ and $\omega$, which are produced at very high interaction energies with the ratio 9:1 and a cross section proportional to the total cross section. Finally, the residuals to the total cross section are fitted by a simple model and treated as statistical multipion production, which is simulated by a QCD string fragmentation model (Andersson et al. 1983). After the decay of all intermediate states, considering basic kinematical relations and accelerator data on rapidity distributions, the resulting distributions of protons, neutrons and pions in the source determine the measurable astrophysical quantities, for example,

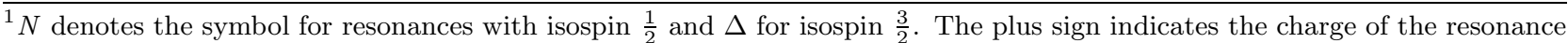
and the bracketed number its (nominal) mass [see Particle Data Group (1996) for a description of the naming scheme of hadrons].
} 


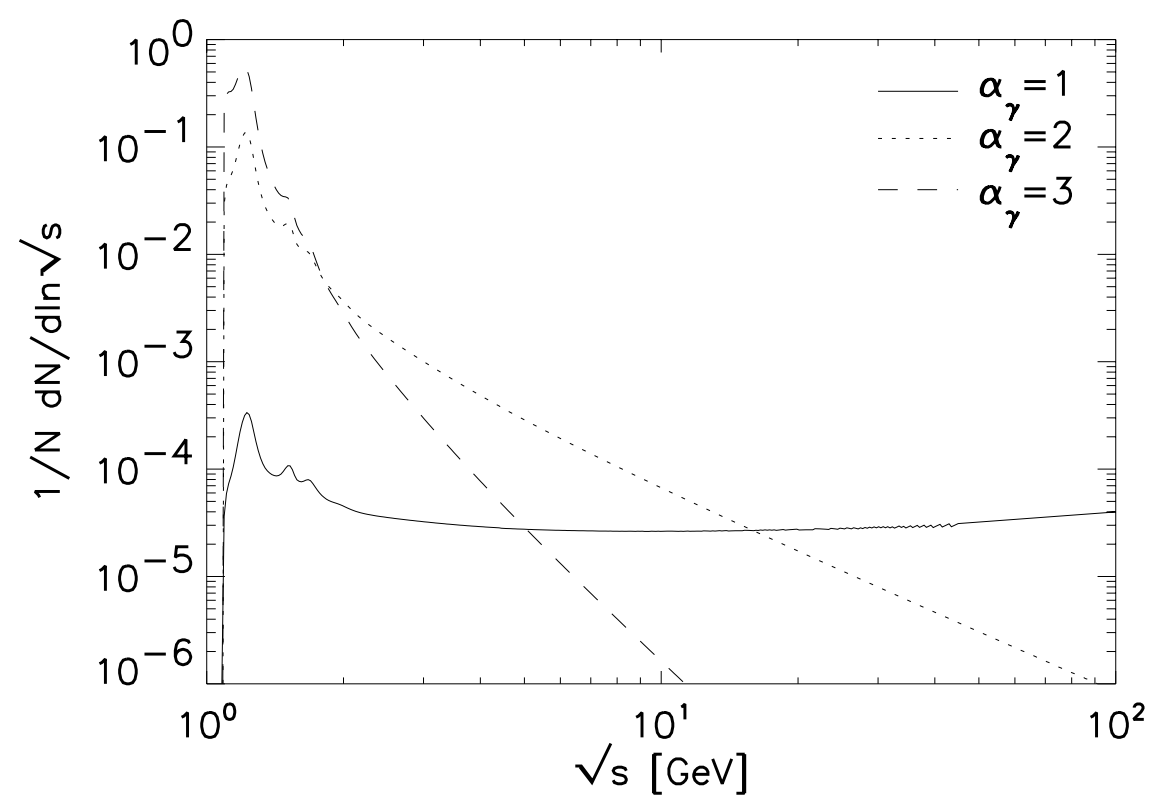

Figure 2-Interaction probability distribution as function of the CMF energy with photon index $\alpha_{\gamma}$ and proton spectrum $n_{E} \sim E^{-2}$.

the cosmic ray, neutrino and $\gamma$-ray emission. Details of the simulation techniques are described in Mücke et al. (1999a).

In the $\Delta$ approximation (Stecker 1973; Gaisser, Halzen \& Stanev 1995), the total cross section is given as $\sigma_{\Delta}=500 \mu$ barn $\Theta\left(\sqrt{s}-m_{\Delta}+\Gamma_{\Delta} / 2\right) \Theta\left(m_{\Delta}+\right.$ $\left.\Gamma_{\Delta} / 2-\sqrt{s}\right)$, where $m_{\Delta}=1 \cdot 232 \mathrm{GeV}$ is the mass and $\Gamma_{\Delta}=0 \cdot 115 \mathrm{GeV}$ is the width of the $\Delta(1232)$ resonance, and $\Theta$ is the Heaviside step function. The $\Delta$ approximation uses the branching ratios of the $\Delta^{+}(1232)$-resonance to determine the number ratio $\pi^{0}$ to $\pi^{+}$of $2: 1$. Photohadronic $\nu$-production is the result of the decay of charged secondary pions $\left(\pi^{+} \rightarrow e^{+} \nu_{\mu} \bar{\nu}_{\mu} \nu_{e}, \pi^{-} \rightarrow e^{-} \nu_{\mu} \bar{\nu}_{\mu} \bar{\nu}_{e}\right)$. Gamma-rays are produced via neutral pion decay $\left(\pi^{0} \rightarrow \gamma \gamma\right)$ and synchrotron/Compton emission from the resulting relativistic leptons. In the $\Delta$ approximation this leads to a ratio of the energy content in $\gamma$-rays to neutrinos $\sum E_{\gamma}: \sum E_{\nu} \equiv \mathcal{E}_{\gamma}: \mathcal{E}_{\nu}=3: 1$. The decay kinematics of this resonance decay predict a nucleon inelasticity of $K_{p}=\Delta E_{p} / E_{p} \approx 0 \cdot 2$.

\section{Astrophysical Applications}

In this section we illustrate the importance of using the complete photohadronic cross section and final state composition and kinematics for a number of astrophysical applications. In particular, we compare the average proton inelasticity in a photopion production $K_{p}$ and the ratio $\mathcal{E}_{\gamma} / \mathcal{E}_{\nu}$ from simulations with SOPHIA to the $\Delta$ approximation. We assume that due to radiative processes, electrons convert all their kinetic energy into photons, and so $e^{ \pm}$are counted in $\mathcal{E}_{\gamma}$. The total neutrino energy $\mathcal{E}_{\nu}$ is the sum of the energies of $\nu_{e}, \nu_{\mu}$ and their antiparticles.

\subsection{Power-law Seed Photon Spectra}

In order to illustrate the contribution of the different interaction processes in various astrophysical environments, we convolve the cross section with proton and seed photon spectra. Proton spectra, as well as non-thermal seed photon spectra, can be well approximated by power laws. Figure 2 shows the distribution of the number of interactions with $\mathrm{CMF}$ energy squared, $s$, for power-law seed photon spectra $n_{\epsilon} \sim \epsilon^{-\alpha_{\gamma}}$ and a proton spectrum $n_{E} \sim E_{p}^{-\alpha_{p}}$ with index $\alpha_{p}=2$. Spectra with $\alpha_{p}=2$ are typical for shock-accelerated particles. Shock acceleration is believed to be the dominant process for acceleration of galactic cosmic rays (Jones \& Ellison 1991). It may also be present in extragalactic particle accelerators (Biermann \& Strittmatter 1987), in particular AGN (e.g. Stecker et al. 1991; Mannheim 1993, Protheroe 1997) and in GRBs (e.g. Waxman 1995; Vietri 1998a,b).

It has been proposed that the high-energy gamma ray emission from blazar jets as observed in the $\mathrm{GeV}$ to $\mathrm{TeV}$ energy range results from photopion production in a proton-electron plasma where the relativistic electrons provide a synchrotron seed photon field for the high-energy protons ('Proton Blazar' model, Mannheim 1993). The synchrotron photon spectrum is described by a power law which is often observed in jets of AGN. Photopion production is followed by electromagnetic cascades reprocessing the injected power of the pion decay products (Mannheim, Krülls \& Biermann 1991). Therefore, Figure 2 may approximate the situation in AGN jets. While for steep photon spectra the prominent resonance region near threshold is the dominating contributor to the interaction rate, the high-energy region of the cross section gains importance for flat 


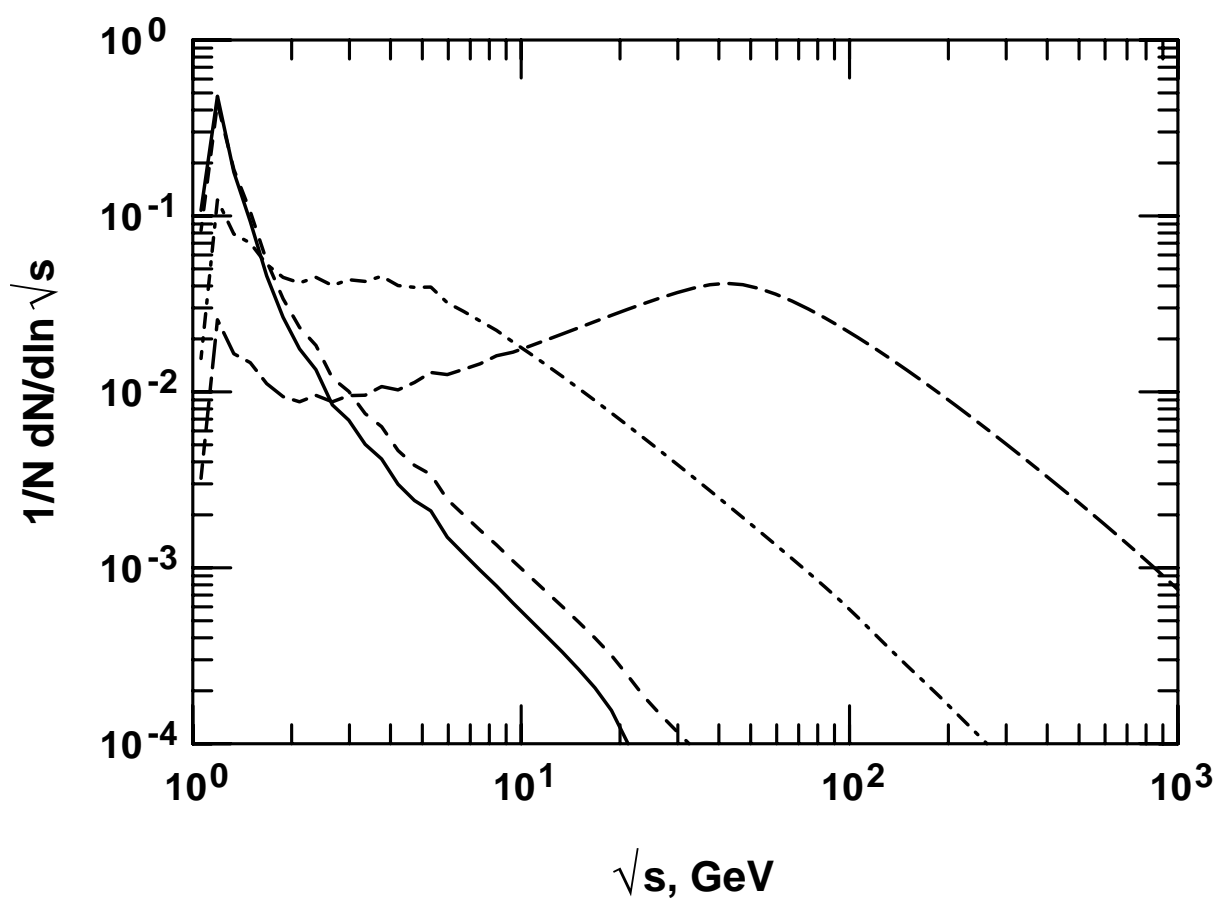

Figure 3-Interaction probability distribution as a function of the CMF energy for a broken power-law seed photon spectra as observed in GRBs, using $n_{\epsilon} \sim \epsilon^{-2 / 3}$ for $10^{-8} \mathrm{eV} \leq \epsilon \leq 1$ $\mathrm{keV}$, and $n_{\epsilon} \sim \epsilon^{-2}$ for $1 \mathrm{keV} \leq \epsilon \leq 100 \mathrm{keV}$ and proton spectrum $n_{E} \sim E^{-\overline{2}}$ with $E_{\min } \leq E \leq 10^{21} \mathrm{eV}$ and different minimum energies $E_{\min }=10^{12} \mathrm{eV}$ (solid line), $10^{14} \mathrm{eV}$ (dashed line), $10^{16} \mathrm{eV}$ (dashed-dotted line) and $10^{18} \mathrm{eV}$ (dashed-dashed line). All quantities are in the fluid frame comoving with a small element of the blast wave region in GRB fireballs.

seed photon spectra. Radio spectra with $\alpha_{\gamma} \approx 1$ ( $F_{\nu} \propto$ const.) up to a break frequency $\nu_{b}$ are, for example, observed in flat-spectrum radio quasars (FSRQs) and BL Lac objects. Such spectra are believed to originate from a superposition of several self-absorbed synchrotron components (Cotton et al. 1980; Shaffer \& Marscher 1979). Above $\nu_{b}$ the spectrum is loss-dominated (i.e. the cooling timescale is shorter than the dynamical time scale) and steepens to $\alpha_{\gamma} \approx 2$. Recent observations of blazars have revealed synchrotron peaks ranging from the optical/IR-band up to UV/soft X-rays, especially in the flaring state (see e.g. Fossati et al. 1998). Thus flat power-law photon spectra exist in AGN jets and would shift the average CMF energy of photopion production towards high values. For protons with energies below $10^{16} \mathrm{eV}$, only the infrared and higher-frequency photons are relevant for photopion production, due to the threshold condition. For blazars with a synchrotron peak at rather low frequencies, the target photon spectrum for photomeson production may be rather steep, and photohadronic interactions would mainly occur near the threshold.

In both flat and steep photon spectra, the simulations with SOPHIA show that the $\gamma$-ray-to- $\nu$ energy ratio is $\mathcal{E}_{\gamma} / \mathcal{E}_{\nu} \approx 1$. The difference with the $\Delta$ approximation $\left(\mathcal{E}_{\gamma} / \mathcal{E}_{\nu}=3\right)$ is due to the contribution of the second resonance and the multipion production regions in the case of flat photon spectra. In steep spectra, the difference is explained by the influence of the immediate threshold region, where the direct channel dominates over the $\Delta$ resonance (Mücke et al. 1999b). Therefore the $\gamma$-ray and neutrino outputs are approximately equal. This may have significant impact on estimates of the predicted neutrino fluxes from AGN jets, which are usually normalised to the observed $\gamma$-ray luminosity.

The absolute $\gamma$-ray and neutrino energy outputs depend on the total proton energy loss $K_{p} E_{p}$. For photon power-law indices $\alpha_{\gamma} \geq 2$, we find $K_{p} \approx 0 \cdot 2$, as in the $\Delta$ approximation. The inelasticity increases rapidly for flatter photon spectra, reaching $K_{p} \approx 0 \cdot 6$ for $\alpha_{\gamma}=1$. This shows that the $\Delta$ approximation underestimates significantly the fractional energy loss of the incident nucleon in environments with flat seed photon spectra, where protons cool much faster.

Proton energy losses, however, limit the maximum possible proton energy that can be achieved in AGN jets (Biermann \& Strittmatter 1987). Setting the timescales for proton acceleration and energy loss equal, Mannheim (1993) obtained cutoff energies of $\sim 10^{16} \mathrm{eV}$. This estimate was based on a mean cross section for photopion production $\left\langle K_{p} \sigma_{p \gamma}\right\rangle=50 \mu \mathrm{barn}$ and a mean inelasticity $\left\langle K_{p}\right\rangle=0 \cdot 25$. Noting that for flat seed photon spectra sopHIA simulations give proton inelasticities about a factor of 2 larger than 


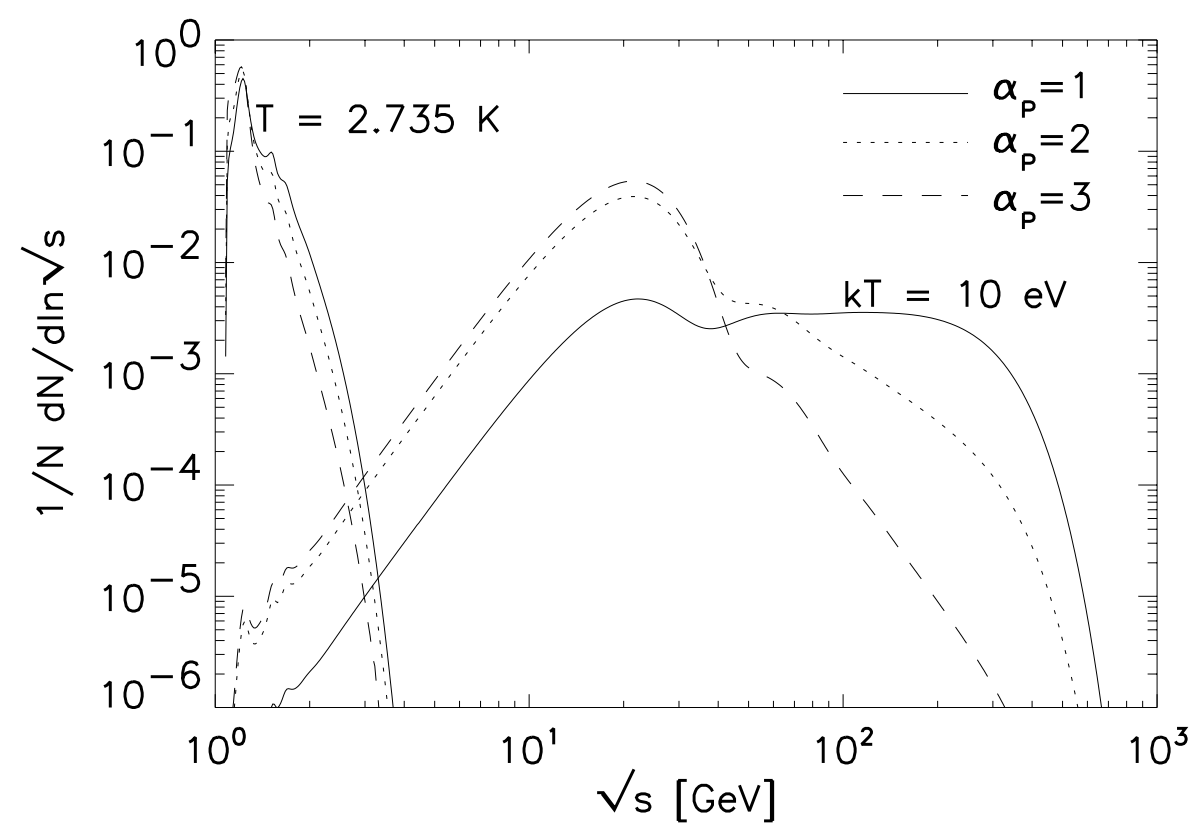

Figure 4-Interaction probability distribution as a function of the CMF energy, for black body seed photon spectra and proton input spectra $n_{E} \sim E_{p}^{-\alpha_{p}}$ in the energy range $E_{p}=10^{6}-10^{12} \mathrm{GeV}$.

those of Mannheim (1993), we expect slightly lower maximum proton energies. Similar corrections could apply to any proton acceleration model where the maximum proton energy is limited by photohadronic interactions. In contrast, models in which Larmor radius constraints and finite acceleration timescales limit the proton energies would not be affected (e.g. proton acceleration in hot spots of radio galaxies, Rachen \& Biermann 1993).

An ideal environment for proton acceleration may be found in gamma-ray bursts that have a broken power-law photon spectrum steepening from $\alpha_{\gamma} \approx 1$ to $\alpha_{\gamma} \approx 2$ at $\mathrm{keV}$ energies (in the comoving frame). Some GRB models involve the acceleration of ultrahigh (energy cosmic rays. Cascades initiated by photopion production follow (Waxman \& Bahcall 1997; Vietri 1998a,b; Böttcher \& Dermer 1998; Rachen \& Meszaros 1998). Figure 3 shows the probability of photoproduction interaction at squared CMF energy $s$ for an $E^{-2}$ proton spectrum with a photon spectrum having $\alpha_{\gamma}=\frac{2}{3}$ below $1 \mathrm{keV}$ and $\alpha_{\gamma}=2$ above that energy. The curves are calculated for different parts of the proton spectrum, extending from minimum energies of $10^{12}-10^{18} \mathrm{eV}$ to a high-energy cutoff of $10^{21} \mathrm{eV}$. While for a proton spectrum with $E_{\min }=10^{12} \mathrm{eV}$ most interactions occur on the $\epsilon^{-2}$-part of the photon field, for higher $E_{\min }$ proton-photon interactions involve the flat part of the seed photon spectrum, leading to higher average $s$ values. Consequently, a $\gamma$-ray-to-neutrino energy ratio of 1 and inelasticities of the order of $0.5-0.7$ are expected for the production of the highest neutrino and secondary photon energies (Mücke et al. 1999b).
Recently, Vietri (1998a,b) has claimed that in situ photoproduced neutrinos in the blast waves associated with GRBs can reach energies up to $\approx 10^{19} \mathrm{eV}$. In order to calculate the maximum neutrino energy, he assumed that individual neutrinos typically carry $5 \%$ of the incident proton energy which is derived from the $\Delta$ approximation. However, SOPHIA simulations show that this value is rather about $1 \%$ due to the increasing neutrino multiplicity constraining the maximum neutrino energies to lower values (Mücke et al. 1999b).

Since the average interaction energies are well beyond the resonance region in GRBs, we expect that detailed calculations with SOPHIA will change significantly the predictions of hadronic GRB models.

\subsection{Thermal Seed Photon Spectra}

By contrast to power-law photon spectra, thermal (blackbody) photon fields are strongly concentrated about a characteristic energy $k T$. This concentration of the seed photons leads to an emphasis of one specific photoproduction energy range. We consider the interactions of protons with different powerlaw spectra with photons from $T=2.73 \mathrm{~K}$ and $k T=10 \mathrm{eV}$ blackbody spectra. The former case is of particular astrophysical interest for cosmic ray (CR) propagation through the CMBR. The latter case may approximately describe the situation in AGN jets, with the accretion disk photons being the target for proton-photon interactions. We show the interaction probability as a function of $s$ in Figure 4. In the case of $\mathrm{CR}$ transport through the CMBR, the $\Delta(1232)$-resonance region plays the dominant role. SOPHIA simulations give a photon-to-neutrino 
energy ratio $\mathcal{E}_{\gamma} / \mathcal{E}_{\nu} \approx 1 \cdot 7$ and inelasticities of $\approx 0 \cdot 2$. We note that the neutrino component dominates directly at the threshold, with $\mathcal{E}_{\gamma} / \mathcal{E}_{\nu} \approx \frac{1}{2}$ due to the dominance of the direct channel.

Recent AGASA measurements seem to indicate an extension of the CR spectrum beyond $10^{20} \mathrm{eV}$ (Takeda et al. 1998). It is generally assumed that acceleration scenarios are limited to maximum energies of $10^{21} \mathrm{eV}$. If the $\mathrm{CR}$ proton spectrum extends above $10^{21} \mathrm{eV}$, one may have to consider so called top-down (TD) models, in which the highest energy cosmic rays are decay products of GUTscale ultraheavy $\left(10^{25} \mathrm{eV}\right)$ particles (e.g. Hill 1983; Sigl, Schramm \& Bhattacharjee 1994; Protheroe \& Stanev 1996). While for TD models the photonto-neutrino energy ratio would reach unity and proton inelasticities can rise up to $0 \cdot 6$, in the case of acceleration scenarios the $\Delta$ approximation describes sufficiently well the proton propagation through the CMBR.

In previous Monte Carlo calculations of cosmic ray pion photoproduction interactions (e.g. Protheroe \& Johnson 1996), an earlier photoproduction event generator based on the observed inclusive cross sections for $p \gamma \rightarrow \pi^{0} p$, and for $p \gamma \rightarrow \pi^{+} n$, together with a semiempirical model for multipion production was used (with equal numbers of $\pi^{+}, \pi^{-}$and $\pi^{0}$ produced in the central region, and charge ratios in the fragmentation regions based on naive quark model arguments; Szabo \& Protheroe 1994). The results from this previous event generator are not too different, and give $\mathcal{E}_{\gamma} / \mathcal{E}_{\nu} \approx 1.6$ and inelasticity $\approx 0 \cdot 2$ at the peak of the resonance, and $\mathcal{E}_{\gamma} / \mathcal{E}_{\nu} \approx 1 \cdot 0$ and inelasticity $\approx 0.55$ in the extreme multipion region.

For photopion production in an isotropic UV radiation field, the high-energy and resonance regions of the cross section are of equal importance (see Figure 4). Thermal radiation peaking in the UV range of the electromagnetic spectrum is observed from some radio-loud AGN (for example 3C 273). Therefore a hadronic blazar model has been proposed in which protons accelerated in some regions of the jet interact with accretion disk photons ('Accretion Disk Proton Blazar' model, Protheroe 1997). In this model the protons, being charged, are isotropised in the jet frame and readily interact with the almost blackbody photons coming from the inner part of the accretion disk near the base of the jet, provided that the disk is sufficiently luminous or the emission region is not too far away from the disk. The model is tuned such that the resulting $\gamma$-rays, which are relativistically beamed along the jet direction, undergo a pair-synchrotron cascade in the accretion disk radiation and jet magnetic field such that the emerging radiation can reproduce features of the observed $\gamma$-ray emission of blazars. The physics implemented in sopHIA allows for a detailed investigation of the parameter space of this model.

\section{Conclusions}

We use our recently developed Monte Carlo code for photohadronic processes (SOPHIA) to compare predictions of astrophysically relevant quantities with the expectations from the widely used ' $\Delta$ approximation'. We discuss proton inelasticities and $\gamma$-ray-to-neutrino energy ratios in the framework of astrophysical environments like AGN, GRBs and for the case of $\mathrm{CR}$ propagation. sopHIA uses the full information of the cross section and the final state particle composition and kinematics of the interaction processes as provided by particle physics. We show that the $\Delta$ approximation is a reasonable approximation only for a restricted set of applications. Several astrophysical cases are discussed:

- Photohadronic interactions of high-energy nucleons in typical radiation fields of GRBs, which may be responsible for the highest-energy $\gamma$-rays and neutrinos, occur mainly at high CMF energies. Nucleon inelasticities of $\sim 0 \cdot 5-0 \cdot 6$ are expected in this case, whereas the $\Delta$ approximation gives only $\approx 0 \cdot 2$. The $\gamma$-ray and neutrino energy outputs are approximately equal.

- High-energy $\gamma$-ray emission from blazar jets may be explainable in terms of photopion production of relativistic protons bathed in a synchrotron or thermal (UV photons from the accretion disk, IRphotons from the molecular torus) radiation field ('Proton Blazar' models). For a flat-spectrum seed photon field, photohadronic interactions with high CMF energies become important. SOPHIA simulations show that protons may cool much faster than in the $\Delta$ approximation because of the growth of the inelasticity $K_{p}$. The $\gamma$-rayto-neutrino energy ratio may approach unity for both flat and steep seed photon spectra, whereas many models use $\mathcal{E}_{\gamma} / \mathcal{E}_{\nu}=3$. This would affect estimates of the predicted neutrino flux.

- Interactions of ultrahigh-energy cosmic rays propagating through the CMBR mainly happen in the region of the $\Delta(1232)$-resonance for energies $<10^{21} \mathrm{eV}$. Above that energy the effects of the second resonant and multiparticle regions increase the fractional energy loss and alter the final shape of the cosmic ray spectrum. Detailed calculations addressing photomeson production in the above mentioned astrophysical environments will be reported in forthcoming publications.

\section{Acknowledgments}

The work of AM and RJP is supported by the Australian Research Council. RE and TS acknowl- 
edge the support by the US Department of Energy under Grant Number DE FG02 01 ER 40626. The work of TS is also supported in part by NASA NAG5-7009. The work of JPR is supported by NASA NAG5-2857. TS thanks the University of Adelaide for hospitality during his visit, which was funded in part by the International Visitor Program of the Special Research Centre for Theoretical Astrophysics, University of Sydney, when this work was started. We acknowledge a helpful discussion with Eli Waxman and Venya Berezinsky.

\section{References}

Andersson, B., et al. 1983, Phys. Rep., 97, 31

Baldini, A., Flaminio, V., Moorhead, W. G., \& Morrison, D. R. O. 1988, in Landold-Börnstein, New Ser., Vol. I/12b, ed. H. Schopper (Berlin: Springer) and references therein Berezinsky, V., \& Gazizov, A. 1993a, Phys. Rev. D, 47, 4206 Berezinsky, V., \& Gazizov, A. 1993b, Phys. Rev. D, 47, 4217

Biermann, P. L., \& Strittmatter, P. A. 1987, ApJ, 322, 643 Böttcher, M., \& Dermer, C. D. 1998, ApJL, 499, L131

Cotton, W. D., Wittels, J. J., Shapiro, I. I., et al. 1980, ApJL, 238, L123

Fossati, G., et al. 1998, MNRAS, 299, 433. Gaisser, T. K., Halzen, F., \& Stanev, T. 1995, Phys. Rep., 258, no.3

Greisen, K. 1966, Phys. Rev. Lett., 16, 748

Hill, C. T. 1983, Nucl. Phys. B, 224, 469

Jones, F. C., \& Ellison, D. C. 1991, Space Sci. Rev., 58, 259
Mannheim, K., Krülls, W. M. \& Biermann, P. L. 1991, A\&A, 251, 723

Mannheim, K. 1993, A\&A, 269, 67

Mücke, A., et al. 1999a, Comput. Phys. Commun., submitted

Mücke, A., et al. 1999b, in preparation

Particle Data Group 1996, Phys. Rev. D, 54, 76

Protheroe R. J., \& Johnson, P. J. 1996, Astroparticle Phys., 4, 253 and erratum 5, 215

Protheroe R. J., \& Stanev, T. 1996, Phys. Rev. Lett., 77, 3708

Protheroe, R. J. 1997, in Accretion Phenomena and Related Outflows, IAU Colloq. 163, Port Douglas, July 1996, ASP Conf. Ser., Vol. 121, ed. D. T. Wickramasinghe et al. (San Francisco: PASP), p. 585

Rachen, J. P. 1996, PhD thesis, MPIfR, Bonn, Germany (http://www.astro.psu.edu/users/jorg/PhD)

Rachen, J. P., \& Biermann, P. L. 1993, A\&A, 272, 161

Rachen J. P., \& Meszaros, P. 1998, Phys. Rev. D, 58, 123005

Shaffer D. B. \& Marscher, A. P. 1979, ApJL, 233, L105

Sigl, G., Schramm, D. N., \& Bhattacharjee, P. 1994. Astroparticle Phys., 2, 401

Stecker, F. W. 1973, A\&SS, 20, 47

Stecker, F. W., et al. 1991, Phys. Rev. Lett., 66, 2697 and erratum 69, 2738 (1992)

Szabo, A. P., and R. J. Protheroe 1994, Astroparticle Phys., 2,375

Takeda, M., et al 1998, Phys. Rev. Lett., 81, 1163

Vietri, M. 1998a, ApJ, 507, 40

Vietri, M. 1998b, Phys. Rev. Lett., 80, 3690

Waxman, E. J. 1995, Phys. Rev. Lett., 75, 386

Waxman, E., \& Bahcall, J. 1997, Phys. Rev. Lett., 78, 2292

Zatsepin, G. T., \& Kuzmin, V. A. 1966, JETP Lett., 4, 78 